\title{
On free massless (pseudo)scalar quantum field theory in 1+1-dimensional space-time
}

\author{
M. Faber* and A. N. Ivanov ${ }^{\dagger \ddagger}$ \\ February 1, 2008 \\ Atominstitut der Österreichischen Universitäten, Arbeitsbereich Kernphysik und \\ Nukleare Astrophysik, Technische Universität Wien, \\ Wiedner Hauptstr. 8-10, A-1040 Wien, Österreich
}

\begin{abstract}
We construct a consistent quantum field theory of a free massless (pseudo)scalar field in 1+1-dimensional space-times free of infrared divergences. We show that in such a quantum field theory (i) a continuous symmetry of (pseudo)scalar field translations is spontaneously broken, (ii) Goldstone bosons appear as quanta of a free massless (pseudo)scalar field and (iii) there is a nonvanishing spontaneous magnetization. In spite of the existence of a spontaneous magnetization the main inequality between vacuum expectation values of certain operators which have been used for the derivation of the Mermin-Wagner-Hohenberg theorem (C. Itzykson and J.-M. Drouffe, STATISTICAL FIELD THEORY, Vol. I, 1989, pp.219-224) is fulfilled.
\end{abstract}

\footnotetext{
${ }^{*}$ E-mail: faber@kph.tuwien.ac.at, Tel.: +43-1-58801-14261, Fax: +43-1-5864203

${ }^{\dagger} \mathrm{E}$-mail: ivanov@kph.tuwien.ac.at, Tel.: +43-1-58801-14261, Fax: +43-1-5864203

${ }^{\ddagger}$ Permanent Address: State Technical University, Department of Nuclear Physics, 195251 St. Petersburg, Russian Federation
} 


\section{Introduction}

As has been noted by Klaiber in his seminal paper [1] devoted to the solution of the massless Thirring model $[2,3]$ within the operator formalism, the main problem of quantum field theories in 1+1-dimensional space-time is the infrared divergence of the two-point Wightman functions of a free massless (pseudo)scalar field $\vartheta(x)$

$$
\begin{aligned}
& D^{(+)}(x)=\langle 0|\vartheta(x) \vartheta(0)| 0\rangle=\frac{1}{2 \pi} \int_{-\infty}^{\infty} \frac{d k^{1}}{2 k^{0}} e^{-i k \cdot x}=-\frac{1}{4 \pi} \ln \left[-\mu^{2} x^{2}+i 0 \cdot \varepsilon\left(x^{0}\right)\right] \\
& D^{(-)}(x)=\langle 0|\vartheta(0) \vartheta(x)| 0\rangle=\frac{1}{2 \pi} \int_{-\infty}^{\infty} \frac{d k^{1}}{2 k^{0}} e^{+i k \cdot x}=-\frac{1}{4 \pi} \ln \left[-\mu^{2} x^{2}-i 0 \cdot \varepsilon\left(x^{0}\right)\right]
\end{aligned}
$$

where $\varepsilon\left(x^{0}\right)$ is the sign function, $x^{2}=\left(x^{0}\right)^{2}-\left(x^{1}\right)^{2}, k \cdot x=k^{0} x^{0}-k^{1} x^{1}, k^{0}=\left|k^{1}\right|$ is the energy of a free massless (pseudo)scalar quantum with momentum $k^{1}$ and $\mu$ is the infrared cut-off reflecting the infrared divergence of the Wightman function (1.1) 1. This was already stated by Klaiber [1]: "If one wants to solve the Thirring model, one has to overcome this problem."

The problem of the infrared divergence of the Wightman function of a free massless (pseudo)scalar field (1.1) has than been reformulated by Coleman [4] as the absence of Goldstone bosons and, correspondingly, of a spontaneously broken continuous symmetry in 1+1-dimensional space-time.

Recently [5] we have shown that Coleman's statement concerning the absence of a spontaneously broken continuous symmetry in 1+1-dimensional space-time is questionable. Indeed, the main distinction of a spontaneously broken continuous symmetry from an unbroken one is in the appearance of massless Goldstone bosons [6]. According to Goldstone's theorem [6], Goldstone bosons accompany the spontaneous breaking of a continuous symmetry. In this connection Coleman [4] argued that in a 1+1-dimensional quantum field theory of a massless (pseudo)scalar field there are no Goldstone bosons. In order to prove this statement Coleman considered a quantum field theory of a free massless (pseudo)scalar field $\vartheta(x)$ with the Lagrangian

$$
\mathcal{L}(x)=\frac{1}{2} \partial_{\mu} \vartheta(x) \partial^{\mu} \vartheta(x),
$$

where $x=\left(x^{0}, x^{1}\right)$ is a 2 -vector. The equation of motion of the $\vartheta$-field reads

$$
\square \vartheta(x)=0 .
$$

The Lagrangian (1.2) is invariant under field translations [5,7]

$$
\vartheta(x) \rightarrow \vartheta^{\prime}(x)=\vartheta(x)-2 \alpha_{\mathrm{A}}
$$

where $\alpha_{\mathrm{A}}$ is an arbitrary parameter. The conserved current associated with these field translations is equal to

$$
j_{\mu}(x)=\partial_{\mu} \vartheta(x) .
$$

\footnotetext{
${ }^{1}$ Further in order to underscore the dependence of the two-point Wightman functions of the scale $\mu$ we will denote $D^{( \pm)}(x) \rightarrow D^{( \pm)}(x ; \mu)$.
} 
The total charge is defined by the time-component of $j_{\mu}(x)[5,7]$

$$
Q\left(x^{0}\right)=\lim _{L \rightarrow \infty} \int_{-L / 2}^{L / 2} d x^{1} \frac{\partial}{\partial x^{0}} \vartheta\left(x^{0}, x^{1}\right),
$$

where $L$ is the volume occupied by the system.

It is well-known that the spontaneous breaking of a continuous symmetry occurs when the ground state of the system is not invariant under the symmetry group $[6,8]$. The ground state of the system described by the Lagrangian (1.2) is not invariant under field translations (1.4) [5]. Therefore, the field-translation symmetry should be spontaneously broken and a Goldstone boson should appear [5].

Therewith, the non-invariance of the ground state of the system can be demonstrated by acting with the operator $\exp \left\{-2 i \alpha_{\mathrm{A}} Q(0)\right\}$ on the vacuum wave function $|0\rangle$, i.e. $\left|\alpha_{\mathrm{A}}\right\rangle=$ $\exp \left\{-2 i \alpha_{\mathrm{A}} Q(0)\right\}|0\rangle[5]$.

For the calculation of $\left|\alpha_{\mathrm{A}}\right\rangle$ we use the expansion of the massless (pseudo)scalar field $\vartheta(x)$ into plane waves [5]

$$
\vartheta(x)=\int_{-\infty}^{\infty} \frac{d k^{1}}{2 \pi} \frac{1}{2 k^{0}}\left(a\left(k^{1}\right) e^{-i k \cdot x}+a^{\dagger}\left(k^{1}\right) e^{i k \cdot x}\right),
$$

where $a\left(k^{1}\right)$ and $a^{\dagger}\left(k^{1}\right)$ are annihilation and creation operators obeying the standard commutation relation

$$
\left[a\left(k^{1}\right), a^{\dagger}\left(q^{1}\right)\right]=(2 \pi) 2 k^{0} \delta\left(k^{1}-q^{1}\right) .
$$

From (1.6) we obtain the total charge operator $Q(0)[5]$

$$
Q(0)=-\frac{i}{2}\left[a(0)-a^{\dagger}(0)\right]
$$

Then, we get the wave function $\left|\alpha_{\mathrm{A}}\right\rangle$

$$
\left|\alpha_{\mathrm{A}}\right\rangle=e^{-2 i \alpha_{\mathrm{A}} Q(0)}|0\rangle=e^{-\alpha_{\mathrm{A}}\left[a(0)-a^{\dagger}(0)\right]}|0\rangle .
$$

For the subsequent mathematical operations with the wave functions $\left|\alpha_{\mathrm{A}}\right\rangle$ it is convenient to use the regularization procedure suggested by Itzykson and Zuber [7]. We define the regularized operator $Q(0)_{\mathrm{R}}$ as follows [5]

$$
Q(0)_{\mathrm{R}}=\left.\lim _{L \rightarrow \infty} \int_{-\infty}^{\infty} d x^{1} \frac{\partial}{\partial x^{0}} \vartheta\left(x^{0}, x^{1}\right)\right|_{x^{0}=0} e^{-\left(x^{1}\right)^{2} / L^{2}} .
$$

The regularized wave function $\left|\alpha_{\mathrm{A}}\right\rangle_{\mathrm{R}}$ reads then

$$
\begin{aligned}
\left|\alpha_{\mathrm{A}}\right\rangle_{\mathrm{R}} & =e^{-2 i \alpha_{\mathrm{A}} Q(0)_{\mathrm{R}}}|0\rangle= \\
& =\lim _{L \rightarrow \infty} \exp \left\{-\frac{\alpha_{\mathrm{A}} L}{2 \sqrt{\pi}} \int_{-\infty}^{\infty} d k^{1}\left[a\left(k^{1}\right)-a^{\dagger}\left(k^{1}\right)\right] e^{-L^{2}\left(k^{1}\right)^{2} / 4}\right\}|0\rangle .
\end{aligned}
$$

The normal-ordered energy operator of the massless (pseudo)scalar field described by the Lagrangian (1.2) is equal to

$$
\begin{aligned}
& \hat{H}\left(x^{0}\right)=\int_{-\infty}^{\infty} d x^{1} \mathcal{H}\left(x^{0}, x^{1}\right)=\int_{-\infty}^{\infty} d x^{1}\left[: \pi\left(x^{0}, x^{1}\right) \dot{\vartheta}\left(x^{0}, x^{1}\right)-\mathcal{L}\left(x^{0}, x^{1}\right):\right]= \\
& =\frac{1}{2} \int_{-\infty}^{\infty} d x:\left[\left(\frac{\partial \vartheta\left(x^{0}, x^{1}\right)}{\partial x^{0}}\right)^{2}+\left(\frac{\partial \vartheta\left(x^{0}, x^{1}\right)}{\partial x^{1}}\right)^{2}\right]:=\frac{1}{2} \int_{-\infty}^{\infty} \frac{d k^{1}}{2 \pi} a^{\dagger}\left(k^{1}\right) a\left(k^{1}\right),
\end{aligned}
$$


where $\pi(x)=\dot{\vartheta}(x)$ is the conjugate momentum and $\dot{\vartheta}(x)$ is the time derivative.

One can easily show that the wave functions $\left|\alpha_{\mathrm{A}}\right\rangle_{\mathrm{R}}$ are eigenfunctions of the energy operator (1.13) for the eigenvalue zero

$$
\hat{H}\left(x^{0}\right)\left|\alpha_{\mathrm{A}}\right\rangle_{\mathrm{R}}=E\left(\alpha_{\mathrm{A}}\right)\left|\alpha_{\mathrm{A}}\right\rangle_{\mathrm{R}}=0 .
$$

This shows that the energy of the vacuum state is infinitely degenerate, and the vacuum state depends on the field translations (1.4). The wave functions of the vacuum state $\left|\alpha_{\mathrm{A}}\right\rangle_{\mathrm{R}}$ and $\left|\alpha_{\mathrm{A}}^{\prime}\right\rangle_{\mathrm{R}}$ are not orthogonal to each other for $\alpha_{\mathrm{A}}^{\prime} \neq \alpha_{\mathrm{A}}$ and the scalar product ${ }_{\mathrm{R}}\left\langle\alpha_{\mathrm{A}}^{\prime} \mid \alpha_{\mathrm{A}}\right\rangle_{\mathrm{R}}$ amounts to [5]

$$
{ }_{\mathrm{R}}\left\langle\alpha_{\mathrm{A}}^{\prime} \mid \alpha_{\mathrm{A}}\right\rangle_{\mathrm{R}}=e^{-\left(\alpha_{\mathrm{A}}^{\prime}-\alpha_{\mathrm{A}}\right)^{2}}
$$

Since the eigenvalue of the wave functions $\left|\alpha_{\mathrm{A}}\right\rangle$ is zero, they can be orthogonalized by any appropriate orthogonalization procedure as used in molecular and nuclear physics.

We would like to emphasize that the results expounded above are not related to the impossibility to determine the two-point Wightman function (1.1) in the infrared region of $\vartheta$-field fluctuations. In fact, the analysis of the non-invariance of the vacuum wave function under the symmetry transformations (1.4) treats the massless (pseudo)scalar field at the tree level. This is an appropriate description, since the massless (pseudo)scalar field $\vartheta(x)$ is free, no vacuum fluctuations are entangled and the quanta of the massless $\vartheta$-field are on-mass shell.

Following the Itzykson-Zuber analysis of the 1+1-dimensional massless (pseudo)scalar field theory of the $\vartheta$-field described by the Lagrangian (1.2) one can show [5] that the translation symmetry (1.4) is spontaneously broken: (i) the ground state is not invariant under the field-translation symmetry, (ii) the energy of the ground state is infinitely degenerated and (iii) Goldstone bosons appear and they are the quanta of the $\vartheta$-field. Hence, all requirements for a continuous symmetry to be spontaneously broken are available in the 1+1-dimensional quantum field theory of a massless (pseudo)scalar field described by the Lagrangian (1.2).

The paper is organized as follows. In section 2 we show that the generating functional of Green functions of a free massless (pseudo)scalar field $\vartheta(x)$ does not depend on the infrared cut-off and the two-point causal and Wightman functions can be made finite in the infrared region. In section 3 we consider the low-frequency quanta of a free massless (pseudo)scalar field as an ensemble which is described by a wave function of a coherent state with a random fluctuating field $\eta\left(k^{1}\right)[9]$. We suggest to treat this random field as a hidden parameter of the theory. We assume that all quantities such as correlation functions defined in the quantum field theory of a free massless (pseudo)scalar field $\vartheta(x)$ should be averaged over this parameter. In this way we derive Wightman functions which are non-singular in the infrared region. In the Conclusion we discuss the relation of the infrared regularized quantum field theory of a free massless (pseudo)scalar field $\vartheta(x)$ to the Mermin-Wagner-Hohenberg (MWH) theorem [10] stating the vanishing of the longrange order and a spontaneous magnetization in two dimensional field theories. We show that in the quantum field theory of the free massless (pseudo)scalar field free of infrared divergences there is a non-vanishing spontaneous magnetization. We demonstrate that in spite of the non-vanishing value of the spontaneous magnetization the main inequality which has been used for the derivation of the MWH theorem is fulfilled. We argue that 
this result cannot be a counterexample to the MWH theorem, since this theorem was formulated only for non-zero temparature $T \neq 0$, whereas a spontaneous magnetization in the quantum field theory of the free massless (pseudo)scalar field is calculated for $T=0$. We accentuate that the infrared regularized quantum field theory of a free massless (pseudo)scalar field $\vartheta(x)$ agrees well with the results obtained in Ref.[5] for the solution of the massless Thirring model with fermion fields quantized in the chirally broken phase.

\section{Generating functional of Green functions of a free massless (pseudo)scalar field}

It is well-known that the solution of a quantum field theory corresponds to the evaluation of any correlation function. In the quantum field theory of a free massless (pseudo)scalar field $\vartheta(x)$ any correlation function can be evaluated by means of the generating functional of Green functions defined by

$Z[J]=\left\langle 0\left|\mathrm{~T}\left(e^{i \int d^{2} x \vartheta(x) J(x)}\right)\right| 0\right\rangle=\int \mathcal{D} \vartheta e^{i \int d^{2} x\left[\frac{1}{2} \partial_{\mu} \vartheta(x) \partial^{\mu} \vartheta(x)+\vartheta(x) J(x)\right]}$,

where $\mathrm{T}$ is a time-ordering operator and $J(x)$ is an external source of the free massless (pseudo)scalar field $\vartheta(x)$.

In terms of $Z[J]$ an arbitrary correlation function of the $\vartheta$-field can be defined as follows

$$
\begin{aligned}
& G\left(x_{1}, \ldots, x_{n} ; y_{1}, \ldots, y_{p}\right)=\left\langle 0\left|F\left(\vartheta\left(x_{1}\right), \ldots, \vartheta\left(x_{n}\right) ; \vartheta\left(y_{1}\right), \ldots, \vartheta\left(y_{p}\right)\right)\right| 0\right\rangle= \\
& =\left.F\left(-i \frac{\delta}{\delta J\left(x_{1}\right)}, \ldots,-i \frac{\delta}{\delta J\left(x_{n}\right)} ;-i \frac{\delta}{\delta J\left(y_{1}\right)}, \ldots,-i \frac{\delta}{\delta J\left(y_{p}\right)}\right) Z[J]\right|_{J=0}
\end{aligned}
$$

As usual in the sine-Gordon, Thirring and $X Y$ models [11-16] one encounters the problem of the evaluation of correlation functions of the following kind

$$
\begin{aligned}
& G\left(x_{1}, \ldots, x_{n} ; y_{1}, \ldots, y_{p}\right)=\left\langle 0\left|\mathrm{~T}\left(\prod_{j=1}^{n} e^{+i \beta \vartheta\left(x_{j}\right)} \prod_{k=1}^{p} e^{-i \beta \vartheta\left(y_{k}\right)}\right)\right| 0\right\rangle= \\
& =\left.\exp \left\{-i \beta \sum_{j=1}^{n} \frac{\delta}{\delta J\left(x_{j}\right)}+i \beta \sum_{k=1}^{p} \frac{\delta}{\delta J\left(y_{k}\right)}\right\} Z[J]\right|_{J=0}
\end{aligned}
$$

Since the path-integral over the $\vartheta$-field (2.1) is Gaussian, it can be evaluated explicitly. The result reads

$$
Z[J]=\lim _{\mu \rightarrow 0} \exp \left\{i \frac{1}{2} \int d^{2} x d^{2} y J(x) \Delta(x-y ; \mu) J(y)\right\}
$$

where $\Delta(x-y ; \mu)$, the causal two-point Green function, obeys the equation

$$
\square \Delta(x-y ; \mu)=\delta^{(2)}(x-y)
$$

and is given by the expression

$$
\Delta(x-y ; \mu)=-\frac{i}{4 \pi} \ln \left[-\mu^{2}(x-y)^{2}+i 0\right]
$$


where $\mu$ is the infrared cut-off that should be taken finally in the limit $\mu \rightarrow 0$.

The presence of the infrared cut-off is related to the infrared problem of a free massless (pseudo)scalar field theory formulated by Klaiber [1] and Coleman [4]. The removal of the infrared cut-off from the Green function $\Delta(x-y ; \mu)$ and its replacement by a finite scale $M$ should solve this infrared problem.

In order to understand the behaviour of $Z[J]$ in the limit $\mu \rightarrow 0$ we suggest to factorize the contribution of the infrared cut-off $\mu$ by introducing a finite arbitrary scale $M$. This yields

$$
\begin{aligned}
Z[J]= & \lim _{\mu \rightarrow 0} \exp \left\{\frac{1}{8 \pi} \int d^{2} x d^{2} y J(x) \ln \left[-\mu^{2}(x-y)^{2}+i 0\right] J(y)\right\}= \\
= & \exp \left\{\frac{1}{8 \pi} \int d^{2} x d^{2} y J(x) \ln \left[-M^{2}(x-y)^{2}+i 0\right] J(y)\right\} \\
& \times \lim _{\mu \rightarrow 0} \exp \left\{-\frac{1}{8 \pi} \ln \frac{M^{2}}{\mu^{2}}\left(\int d^{2} x J(x)\right)^{2}\right\}=\lim _{\mu \rightarrow 0}\left(\frac{\mu}{M}\right)^{\frac{1}{4 \pi}\left(\int d^{2} x J(x)\right)^{2}} \\
& \times \exp \left\{\frac{1}{8 \pi} \int d^{2} x d^{2} y J(x) \ln \left[-M^{2}(x-y)^{2}+i 0\right] J(y)\right\} .
\end{aligned}
$$

Since the power of the ratio $\mu / M$ is always positive, so for any arbitrary external source $J(x)$ with a non-vanishing $1+1$-dimensional volume integral

$$
\int d^{2} x J(x) \neq 0
$$

the generating functional $Z[J]$ vanishes in the limit $\mu \rightarrow 0$, i.e. $Z[J]=0$.

In the Schwinger formulation of quantum field theory [17] the generating functional of Green functions $Z[J]$ given by (2.4) defines the amplitude of the vacuum-vacuum transition $\left\langle 0_{+} \mid 0_{-}\right\rangle^{J}$, i.e. $Z[J]=\left\langle 0_{+} \mid 0_{-}\right\rangle^{J}$. The vanishing of $Z[J]$ corresponds to the vanishing of the amplitude of the vacuum-vacuum transition $\left\langle 0_{+} \mid 0_{-}\right\rangle^{J}=0$. According to Schwinger [17] a quantum field theory with $\left\langle 0_{+} \mid 0_{-}\right\rangle^{J}=0$ has no meaning.

In order to make a quantum field theory of a free massless (pseudo)scalar field $\vartheta(x)$ meaningful we have to get a non-vanishing generating functional of Green functions $Z[J]$. This can be obtained by imposing the constraint

$$
\int d^{2} x J(x)=0
$$

We would like to emphasize that due to this constraint $Z[J]$ becomes invariant under the $\vartheta$-field translations (1.4) as well as the Lagrangian (1.2).

For the massless pseudoscalar field $\vartheta(x)$ the constraint (2.9) is fulfilled automatically. Indeed, due to the conservation of parity the external source of the field $\vartheta(x)$ should obey the relation $J\left(x^{0}, x^{1}\right)=-J\left(x^{0},-x^{1}\right)$. For a scalar $\vartheta$-field, when $J\left(x^{0}, x^{1}\right)=J\left(x^{0},-x^{1}\right)$, the relation (2.2) can be fulfilled for a rather broad class of analytical and generalized functions [18].

Recall that the constraint (2.9) has been implicitly used for the evaluation of correlation functions (2.3) which can be transcribed as follows

$$
G\left(x_{1}, \ldots, x_{n} ; y_{1}, \ldots, y_{p}\right)=\left\langle 0\left|\mathrm{~T}\left(e^{i \int d^{2} x \vartheta(x) J\left(x ; x_{1}, \ldots, x_{n} ; y_{1} \ldots, y_{p}\right)}\right)\right| 0\right\rangle
$$


where the current $J(x)$ is defined by

$$
J\left(x ; x_{1}, \ldots, x_{n} ; y_{1} \ldots, y_{p}\right)=\beta \sum_{j=1}^{n} \delta^{(2)}\left(x-x_{j}\right)-\beta \sum_{k=1}^{p} \delta^{(2)}\left(x-y_{k}\right) .
$$

Substituting (2.11) in (2.9) we obtain

$$
\int d^{2} x J\left(x ; x_{1}, \ldots, x_{n} ; y_{1} \ldots, y_{p}\right)=\beta(n-p) .
$$

It is well-known [11-16] that for $n=p$ the correlation functions (2.10) do not depend on the infrared cut-off, in turn, for $n \neq p$ the dependence of the correlation functions on $\mu$ leads to their vanishing in the limit $\mu \rightarrow 0$. Unfortunately, nobody made an attempt to relate this result with the vanishing of $Z[J]$ in the limit $\mu \rightarrow 0$ for an arbitrary defined external source $J(x)$.

As has been shown in [16] the constraint (2.9) has turned out to be rather useful for the proof of non-perturbative renormalizability of the sine-Gordon model described by the Lagrangian $[5,11,16]$

$$
\mathcal{L}(x)=\frac{1}{2} \partial_{\mu} \vartheta(x) \partial^{\mu} \vartheta(x)+\frac{\alpha}{\beta^{2}}(\cos \beta \vartheta(x)-1),
$$

where $\alpha$ and $\beta$ are parameters of the model $[5,11,16]$. The main peculiarity of the sineGordon model is the existence of soliton solutions having the properties of fermions $[19,20]$ (see also [5]).

Hence, in order to make the quantum field theory of a free massless (pseudo)scalar field meaningful the constraint (2.9) should be imposed. According to this constraint the generating functional of Green functions $Z[J]$ reads

$$
Z[J]=\exp \left\{\frac{1}{8 \pi} \int d^{2} x d^{2} y J(x) \ln \left[-M^{2}(x-y)^{2}+i 0\right] J(y)\right\} .
$$

In this form the generating functional of Green functions $Z[J]$ is well-defined. Thus, the constraint (2.2) provides the independence of the generating functional of Green functions of the free massless (pseudo)scalar field $\vartheta(x)$ of the infrared cut-off. This makes the quantum field theory described by $Z[J]$ in (2.14) meaningful.

Of course, a certain cautiousness is needed because constraints on the external sources $J(x)$ may lead to a loss of information about the response of the quantum system. Therefore, one has to be convinced that in the case of the free massless (pseudo)scalar quantum field theory, defined in 1+1-dimensional space-time, there is no a loss of information about the response of the free massless (pseudo)scalar field to the external perturbations restricted by the constraint (2.9).

The physical meaning of the constraint (2.9) can be easily understood in the momentum representation, where the (pseudo)scalar field $\vartheta(x)$ and the external source $J(x)$ are expressed by their Fourier transforms $\tilde{\vartheta}(k)$ and $\tilde{J}(k)$

$$
\vartheta(x)=\int \frac{d^{2} k}{(2 \pi)^{2}} \tilde{\vartheta}(k) e^{-i k \cdot x} \quad, \quad J(x)=\int \frac{d^{2} k}{(2 \pi)^{2}} \tilde{J}(k) e^{-i k \cdot x} .
$$


By making a change of variables $\vartheta(x) \rightarrow \tilde{\vartheta}(k)$ the generating functional of Green function acquires the form

$$
Z[J]=\int \mathcal{D} \tilde{\vartheta} \exp \left\{i \int \frac{d^{2} k}{(2 \pi)^{2}}\left[\frac{1}{2}\left(k^{2}+i 0\right) \tilde{\vartheta}(k) \tilde{\vartheta}(-k)+\tilde{\vartheta}(k) \tilde{J}(-k)\right]\right\}
$$

Integrating over the $\tilde{\vartheta}$-field we arrive at the expression

$$
\begin{aligned}
Z[J] & =\exp \left\{-i \frac{1}{2} \int \frac{d^{2} k}{(2 \pi)^{2}} \frac{\tilde{J}(k) \tilde{J}(-k)}{k^{2}+i 0}\right\}= \\
& =\exp \left\{-i \frac{1}{2} \int d^{2} x d^{2} y J(x) \int \frac{d^{2} k}{(2 \pi)^{2}} \frac{e^{i k(x-y)}}{k^{2}+i 0} J(y)\right\} .
\end{aligned}
$$

Integrating over $k^{0}$ we reduce the exponent to the form

$$
\begin{aligned}
& Z[J]=\exp \left\{-\frac{1}{4 \pi} \int_{-\infty}^{\infty} \frac{d k^{1}}{2\left|k^{1}\right|} \tilde{J}\left(-\left|k^{1}\right|, k^{1}\right) \tilde{J}\left(\left|k^{1}\right|,-k^{1}\right)\right\}= \\
& =\exp \left\{-\frac{1}{4 \pi} \int d^{2} x d^{2} y J(x)\left[\theta\left(x^{0}-y^{0}\right) \int_{-\infty}^{\infty} \frac{d k^{1}}{2\left|k^{1}\right|} e^{-i\left|k^{1}\right|\left(x^{0}-y^{0}\right)+i k^{1}\left(x^{1}-y^{1}\right)}\right.\right. \\
& +\theta\left(y^{0}-x^{0}\right) \int_{-\infty}^{\infty} \frac{d k^{1}}{2\left|k^{1}\right|} e^{\left.\left.+i\left|k^{1}\right|\left(x^{0}-y^{0}\right)-i k^{1}\left(x^{1}-y^{1}\right)\right] J(y)\right\} .}
\end{aligned}
$$

Substituting the external source $J(x)$ defined by the momentum integral into (2.15) we obtain $\tilde{J}(0)=0$. Hence, the constraint (2.9) is equivalent to the vanishing of the Fourier transform of the external source for zero 2-momentum $k=0$. Due to this the momentum integrals become convergent in the infrared region $k \rightarrow 0$. As result the generating functional $Z[J]$ does not depend on the infrared cut-off $\mu$. This makes $Z[J]$ well-defined.

It is obvious that in $n \geq 3$ dimensional space-time, where the generating functional of Green functions $Z[J]$ is defined by

$$
Z[J]=\exp \left\{-i \frac{1}{2} \int \frac{d^{n} k}{(2 \pi)^{2}} \frac{\tilde{J}(k) \tilde{J}(-k)}{k^{2}+i 0}\right\},
$$

the problem of the ill-definition of the momentum integral in the infrared region does not appear due to the contribution of the measure $d^{n} k \propto k^{n-1} d k$.

In order to clarify the physical meaning of the constraint $\tilde{J}(0)=0$ let us consider the product $\tilde{\vartheta}(k) \tilde{J}(-k)$ in $(2.16)$. Due to $\tilde{J}(0)=0$ we get $\tilde{\vartheta}(0) \tilde{J}(0)=0$. This means that the zero-mode configuration $\tilde{\vartheta}(0)$ of the $\vartheta$-field does not couple to an external source. In other word setting $\tilde{J}(0)=0$ we do not excite the zero-mode configuration of the $\vartheta$-field. As a result this configuration does not contribute to $Z[J]$ or differently to the amplitude of the vacuum-vacuum transition $\left\langle 0_{+} \mid 0_{-}\right\rangle^{J}$.

Such a treatment of the contribution of the zero-mode configuration of the free massless (pseudo)scalar field is equivalent to some extent to the procedure suggested by Hasenfratz [21] $]$ to treat the zero-mode configuration of self-coupled massless scalar fields in lattice $\sigma$-models with $O(N)$ symmetry defined in one and two dimensions in finite and infinite volumes.

\footnotetext{
${ }^{2}$ We are grateful to Oleg Borisenko for calling our attention to Hasenfratz's paper [21].
} 
The role of the zero-mode configuration of the $\vartheta$-field can be understood by analysing a mechanical analogy of a massless (pseudo)scalar field. According to [22] the continuous system described by the Lagrangian (1.2) is equivalent to a one-dimensional chain of $N$ harmonic oscillators with equal masses, equal equilibrium separations and a potential energy taking into account only nearest neighbors. Their motion can be described by displacements $q_{i}(i=1, \ldots, N)$ which couple to the external sources as $\sum_{i=1}^{N} q_{i} J_{i}$. In the representation of normal coordinates this system reduces to the set of $N-1$ decoupled normal non-zero frequency mode configurations and one zero-mode configuration proportional to the displacement of the center of mass of $N$ coupled harmonic oscillators $Q_{0} \propto \sum_{i=1}^{N} q_{i}$. The coupling of this zero-mode configuration to the external sources is proportional to $Q_{0} \sum_{i=1}^{N} J_{i}$. Since at $J_{i}=0(i=1, \ldots, N)$ the zero-mode configuration does not affect the evolution of the system, one can exclude a motion of the center of mass even for $J_{i} \neq 0(i=1, \ldots, N)$ by the constraint $\sum_{i=1}^{N} J_{i}=0$ which corresponds to (2.9).

This clarifies the physical meaning of the constraint (2.9), that is, the removal of the zero-mode configuration related to the motion of the center of mass of $N$ coupled harmonic oscillators, which in the continuous limit and at $N \rightarrow \infty$ are described by the Lagrangian (1.2). For a free quantum system the exclusion of a collective motion of a system as a structureless configuration does not affect the evolution of the system caused by a relative motion in it and, of course, does not lead to a loss of an important information about the response to perturbations induced by external sources.

For a system of coupled quantum fields such an exclusion would not be innocent. However, as has been shown by Hasenfratz [21] that the exclusion of the zero-mode configuration of massless self-coupled scalar fields in one and two dimensions in finite and infinite volumes described by the $\sigma$-models with $O(N)$ internal symmetry allows to construct a self-consistent and well-defined perturbation theory.

The inclusion of a finite scale $M$ instead of the infinitesimal scale $\mu$ leads to Fourier transform of the Wightman function free of infrared divergences. In order to confirm this statement we suggest to transcribe the r.h.s. of (2.14) in the form

$$
Z[J]=\exp \left\{\frac{i}{2} \int d^{2} x d^{2} y J(x) \Delta(x-y ; M) J(y)\right\}
$$

where $\Delta(x-y ; M)$ is given by (2.6) with the replacement $\mu \rightarrow M$ and obeys the equation (2.5).

Now let us show that due to the finite scale $M$ the Wightman functions (1.1) become convergent in the infrared region, $k^{1} \rightarrow 0$. As the causal Green function $\Delta(x ; M)$ is related to the Wightman functions $D^{( \pm)}(x ; M)$ by the standard relation [14]

$$
\Delta(x ; M)=i \theta\left(+x^{0}\right) D^{(+)}(x ; M)+i \theta\left(-x^{0}\right) D^{(-)}(x ; M),
$$

the Wightman functions $D^{( \pm)}(x ; M)$ are equal to

$$
D^{( \pm)}(x ; M)=-\frac{1}{4 \pi} \ln \left[-M^{2} x^{2} \pm i 0 \cdot \varepsilon\left(x^{0}\right)\right] .
$$

Since the r.h.s. of (2.22) can be treated as a limit

$$
D^{( \pm)}(x ; M)=\frac{1}{2 \pi} \lim _{\mu \rightarrow 0}\left(K_{0}\left(\mu \sqrt{-x^{2} \pm i 0 \cdot \varepsilon\left(x^{0}\right)}\right)-K_{0}\left(\mu \lambda_{\mathrm{M}}\right)\right),
$$


where we have denoted $\lambda_{\mathrm{M}}=1 / M$, the Fourier transforms of Wightman functions are defined by (see Appendix C of Ref.[16])

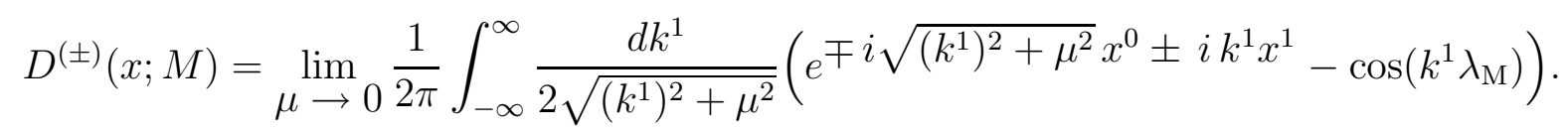

Here we have used the integral representations of the McDonald function $K_{0}(z)$ [23].

Taking the limit $\mu \rightarrow 0$ we obtain

$$
D^{( \pm)}(x ; M)=\frac{1}{2 \pi} \int_{-\infty}^{\infty} \frac{d k^{1}}{2 k^{0}}\left(e^{\mp i k \cdot x}-\cos \left(k^{1} \lambda_{\mathrm{M}}\right)\right) .
$$

Thus, the Wightman functions (2.25) are obviously convergent in the infrared region $k^{1} \rightarrow 0$. This solves the infrared problem of the free massless (pseudo)scalar field theory pointed out by Klaiber [1] and Coleman [4].

\section{Coherent states of low-frequency quanta of a free massless (pseudo)scalar field}

The knowledge of the generating functional of Green functions $Z[J]$ is sufficient for the evaluation of any correlation function in the quantum field theory under consideration. According to (2.2) and (2.3) the evaluation of a correlation function reduces to the calculation of functional derivatives of $Z[J]$ with respect to the external source $J(x)$. One can easily show that the use of the constraint (2.9) during the variation with respect to the external source $J(x)$ does not affect the final result. Therefore, the analysis of Wightman functions in terms of vacuum expectation values of the (pseudo)scalar field $\vartheta(x)$ is no more required.

Nevertheless, to make our consideration of the infrared divergences in the free massless (pseudo)scalar field theory more complete we suggest a variant of the quantum field theory of the free massless (pseudo)scalar field reproducing the two-point Wightman functions (2.25) by means of the redefinition of the (pseudo)scalar field $\vartheta(x)$.

For the derivation of the term $\cos \left(k^{1} \lambda_{\mathrm{M}}\right)$ in (2.25) at the level of the redefinition of a quantum field $\vartheta(x)$ we would like to notice that the wave function $\left|\alpha_{\mathrm{A}}\right\rangle_{\mathrm{R}}(1.12)$ of the vacuum state looks like a wave function of a canonical coherent state produced by a unitary transformation from a fiducial vacuum state $|0\rangle$ [9]. Following this similarity we suggest to describe collective states of low-frequency quanta of the free massless (pseudo)scalar field $\vartheta(x)$, defined by the Lagrangian (1.2), in terms of coherent states [9].

A collective state of low-frequency quanta of the massless scalar field $\vartheta(x)$ we suggest to represent by a wave function

$$
|\eta\rangle=e^{\hat{Q}[\eta]}|0\rangle .
$$

The operator $\hat{Q}[\eta]$ is defined by

$$
\hat{Q}[\eta]=\int_{-\infty}^{\infty} \frac{d k^{1}}{2 \pi} \frac{\eta\left(k^{1}\right)}{2 k^{0}}\left[a\left(k^{1}\right)-a^{\dagger}\left(k^{1}\right)\right]
$$


where $\eta\left(k^{1}\right)$ is an arbitrary function which we treat as a random field variable. The $\eta\left(k^{1}\right)-$ field is concentrated in the infrared region close to $k^{1} \rightarrow 0$ and is almost zero everywhere for finite momenta. For example, in the case of the vacuum wave functions $\left|\alpha_{\mathrm{A}}\right\rangle_{\mathrm{R}}$ the $\eta\left(k^{1}\right)$-field is defined by Gaussian-like functions

$$
\eta_{\alpha_{\mathrm{A}}}\left(k^{1}\right)=-2 \sqrt{\pi} \alpha_{\mathrm{A}} L\left|k^{1}\right| e^{-L^{2}\left(k^{1}\right)^{2} / 4},
$$

where the spatial volume of the system $L$ should tend to infinity, $L \rightarrow \infty$.

The wave function $|\eta\rangle$ is normalized to unity

$$
\langle\eta \mid \eta\rangle=1 .
$$

The wave function (3.1) is constructed in complete analogy to the vacuum wave functions $\left|\alpha_{\mathrm{A}}\right\rangle_{\mathrm{R}}$ related to field translations (1.4) caused by chiral rotations of massless Thirring fermions [5].

The operators of annihilation and creation obey the relations

$$
\begin{aligned}
& e^{\hat{Q}[\eta]} a\left(k^{1}\right) e^{-\hat{Q}[\eta]}=a\left(k^{1}\right)+\eta\left(k^{1}\right), \\
& e^{\hat{Q}[\eta]} a^{\dagger}\left(k^{1}\right) e^{-\hat{Q}[\eta]}=a^{\dagger}\left(k^{1}\right)+\eta\left(k^{1}\right) .
\end{aligned}
$$

This implies that the wave function $|\eta\rangle$ is the eigenfunction of the annihilation operator $a\left(k^{1}\right)$ with the eigenvalue $\eta\left(k^{1}\right)[9]$

$$
a\left(k^{1}\right)|\eta\rangle=\eta\left(k^{1}\right)|\eta\rangle .
$$

Using the relation

$$
e^{A+B}=e^{-\frac{1}{2}[A, B]} e^{A} e^{B}
$$

one can reduce the wave function $|\eta\rangle$ of (3.1) to the standard form of a coherent state [9]

$$
|\eta\rangle=\exp \left\{-\frac{1}{2 \pi} \int_{-\infty}^{\infty} \frac{d k^{1}}{2 k^{0}} \frac{1}{2} \eta^{2}\left(k^{1}\right)\right\} \exp \left\{\int_{-\infty}^{\infty} \frac{d k^{1}}{2 \pi} \frac{\eta\left(k^{1}\right)}{2 k^{0}} a^{\dagger}\left(k^{1}\right)\right\}|0\rangle .
$$

The completeness condition can be represented by a path integral over the $\eta$-field [9]

$$
\hat{1}=\int \mathcal{D} \eta|\eta\rangle\langle\eta|,
$$

where $\hat{1}$ is a unit operator. Using the completeness condition and the normalization of the fiducial vacuum state wave function $\langle 0 \mid 0\rangle=1$ we obtain

$$
\langle 0 \mid 0\rangle=\int \mathcal{D} \eta\langle 0 \mid \eta\rangle\langle\eta \mid 0\rangle=\int \mathcal{D} \eta \exp \left\{-\frac{1}{2 \pi} \int_{-\infty}^{\infty} \frac{d k^{1}}{2 k^{0}} \eta^{2}\left(k^{1}\right)\right\}=1 .
$$

The exponent of the $c$-number factor in $(\sqrt{3.10})$ is related to the number of quanta of the massless (pseudo)scalar $\vartheta$-field in the $|\eta\rangle$ state. In terms of creation and annihilation operators the operator $\hat{N}$ of the number of quanta reads

$$
\hat{N}=\frac{1}{2 \pi} \int_{-\infty}^{\infty} \frac{d k^{1}}{2 k^{0}} a^{\dagger}\left(k^{1}\right) a\left(k^{1}\right) .
$$


Using (3.6) we obtain

$$
N[\eta]=\langle\eta|\hat{N}| \eta\rangle=\frac{1}{2 \pi} \int_{-\infty}^{\infty} \frac{d k^{1}}{2 k^{0}} \eta^{2}\left(k^{1}\right) .
$$

The energy of the state $|\eta\rangle$ is equal to

$$
E[\eta]=\langle\eta|\hat{H}| \eta\rangle=\frac{1}{2} \int_{-\infty}^{\infty} \frac{d k^{1}}{2 \pi} \eta^{2}\left(k^{1}\right)
$$

The action of the creation operator $a^{\dagger}\left(k^{1}\right)$ on the state $|\eta\rangle$ can be obtained by using (3.8) and reads

$$
a^{\dagger}\left(k^{1}\right)|\eta\rangle=\exp \left\{-\frac{1}{2 \pi} \int_{-\infty}^{\infty} \frac{d q^{1}}{2 q^{0}} \frac{1}{2} \eta^{2}\left(q^{1}\right)\right\} \exp \left\{\frac{1}{2 \pi} \int_{-\infty}^{\infty} \frac{d q^{1}}{2 q^{0}} \eta\left(q^{1}\right) a^{\dagger}\left(q^{1}\right)\right\} a^{\dagger}\left(k^{1}\right)|0\rangle .
$$

The r.h.s. of this relation can be rewritten in the form of a variational derivative with respect to $\eta\left(k^{1}\right)$

$$
a^{\dagger}\left(k^{1}\right)|\eta\rangle=\left(\eta\left(k^{1}\right)+\frac{\delta}{\delta \eta\left(k^{1}\right)}\right)|\eta\rangle
$$

where we have used the definition

$$
\frac{\delta \eta\left(q^{1}\right)}{\delta \eta\left(k^{1}\right)}=(2 \pi) 2 k^{0} \delta\left(k^{1}-q^{1}\right)
$$

Since $\eta\left(k^{1}\right)$ is an auxiliary intrinsic, some kind of hidden, parameter of a free massless (pseudo)scalar field theory, all correlation functions should be averaged over the $\eta$-field fluctuations. Such an average we represent in the form of the path-integral over the $\eta$-field fluctuations normalized by the condition (3.10).

Using the random $\eta$-field we introduce instead of $\vartheta(x)$ a new quantum field $\vartheta(x ; \eta)$ defined by

$$
\begin{aligned}
\vartheta(x ; \eta) & =\frac{1}{2 \pi} \int_{-\infty}^{\infty} \frac{d k^{1}}{2 k^{0}}\left(a\left(k^{1}\right) e^{-i k \cdot x}+a^{\dagger}\left(k^{1}\right) e^{i k \cdot x}\right) \\
& +\frac{1}{2 \pi} \int_{-\infty}^{\infty} d k^{1} \eta\left(k^{1}-\pi M\right) \sqrt{\frac{\cos \left(\left(k^{1}-\pi M\right) \lambda_{\mathrm{M}}\right)}{2 k^{0}\left|k^{1}-\pi M\right|}}\left[a\left(k^{1}\right)+a^{\dagger}\left(k^{1}\right)\right] .
\end{aligned}
$$

Since the $\eta\left(k^{1}\right)$ is tangible only in the region of momenta $k^{1}$ comeasurable with zero, the shift of the argument $k^{1} \rightarrow k^{1}-\pi M$ leads to a concentration of the integrand around the momenta $k^{1} \simeq \pi M$, where $\cos \left(\left(k^{1}-\pi M\right) \lambda_{\mathrm{M}}\right)$ is positive. The contribution of the constant term in the definition of the $\vartheta$-field demonstrates the fact that the $\vartheta$-field can be excited above the background by quanta with momenta of order of the scale $M$.

Averaging over the $\eta$-field fluctuations we obtain the quantum field $\vartheta(x)$ defined by (1.7)

$$
\langle\vartheta(x ; \eta)\rangle=\int \mathcal{D} \eta \vartheta(x ; \eta) \exp \left\{-\frac{1}{2 \pi} \int_{-\infty}^{\infty} \frac{d k^{1}}{2 k^{0}} \eta^{2}\left(k^{1}\right)\right\}=\vartheta(x)
$$


Due the constraint (2.9) the additional contribution in (3.17), independing on space-time coordinates and containing the random $\eta\left(k^{1}\right)$-field, does not change the generating functional of Green functions (2.20). However, this contribution turns out to be important for the derivation of the term $\cos \left(k^{1} \lambda_{\mathrm{M}}\right)$ in the regularized two-point Wightman functions (2.25), represented in terms of vacuum expectation values of the products of the $\vartheta(x ; \eta)$-fields and averaged over the random $\eta$-field fluctuations. The regularized twopoint Wightman functions $D^{( \pm)}(x ; M)$ we define as follows

$$
\begin{aligned}
& D^{(+)}(x ; M)= \\
& =\int \mathcal{D} \eta\langle 0|\vartheta(x ; \eta) \vartheta(0 ; \eta)| 0\rangle \exp \left\{-\frac{1}{2 \pi} \int_{-\infty}^{\infty} \frac{d k^{1}}{2 k^{0}} \eta^{2}\left(k^{1}\right)\right\}=\frac{1}{2 \pi} \int_{-\infty}^{\infty} \frac{d k^{1}}{2 k^{0}} e^{-i k \cdot x} \\
& +\frac{1}{2 \pi} \int_{-\infty}^{\infty} d k^{1} \sqrt{\frac{\cos \left(\left(k^{1}-\pi M\right) \lambda_{\mathrm{M}}\right)}{2 k^{0}\left|k^{1}-\pi M\right|}} \frac{1}{2 \pi} \int_{-\infty}^{\infty} d q^{1} \sqrt{\frac{\cos \left(\left(q^{1}-\pi M\right) \lambda_{\mathrm{M}}\right)}{2 q^{0}\left|q^{1}-\pi M\right|}} \\
& \times \int \mathcal{D} \eta \eta\left(k^{1}-\pi M\right) \eta\left(q^{1}-\pi M\right) \exp \left\{-\frac{1}{2 \pi} \int_{-\infty}^{\infty} \frac{d p^{1}}{2 p^{0}} \eta^{2}\left(p^{1}\right)\right\} \\
& D^{(-)}(x ; M)= \\
& =\int \mathcal{D} \eta\langle 0|\vartheta(0 ; \eta) \vartheta(x ; \eta)| 0\rangle \exp \left\{-\frac{1}{2 \pi} \int_{-\infty}^{\infty} \frac{d k^{1}}{2 k^{0}} \eta^{2}\left(k^{1}\right)\right\}=\frac{1}{2 \pi} \int_{-\infty}^{\infty} \frac{d k^{1}}{2 k^{0}} e^{+i k \cdot x} \\
& +\frac{1}{2 \pi} \int_{-\infty}^{\infty} d k^{1} \sqrt{\frac{\cos \left(\left(k^{1}-\pi M\right) \lambda_{\mathrm{M}}\right)}{2 k^{0}\left|k^{1}-\pi M\right|}} \frac{1}{2 \pi} \int_{-\infty}^{\infty} d q^{1} \sqrt{\frac{\cos \left(\left(q^{1}-\pi M\right) \lambda_{\mathrm{M}}\right)}{2 q^{0}\left|q^{1}-\pi M\right|}} \\
& \times \int \mathcal{D} \eta \eta\left(k^{1}-\pi M\right) \eta\left(q^{1}-\pi M\right) \exp \left\{-\frac{1}{2 \pi} \int_{-\infty}^{\infty} \frac{d p^{1}}{2 p^{0}} \eta^{2}\left(p^{1}\right)\right\} .
\end{aligned}
$$

For the integration over the $\eta$-field fluctuations we use the auxiliary integrals

$$
\begin{aligned}
& \int \mathcal{D} \eta \exp \left\{-\frac{1}{2 \pi} \int_{-\infty}^{\infty} \frac{d p^{1}}{2 p^{0}} \eta^{2}\left(p^{1}\right)+\frac{1}{\pi} \int_{-\infty}^{\infty} \frac{d p^{1}}{2 p^{0}} f\left(p^{1}\right) \eta\left(p^{1}\right)\right\}= \\
& =\exp \left\{\frac{1}{2 \pi} \int_{-\infty}^{\infty} \frac{d p^{1}}{2 p^{0}} f^{2}\left(p^{1}\right)\right\}
\end{aligned}
$$

The integration of the quadratic terms in the $\eta$-field in (3.19) we perform with the help of a formula which can be derived from Eq. (3.20)

$$
\begin{aligned}
& \int \mathcal{D} \eta \exp \left\{-\frac{1}{2 \pi} \int_{-\infty}^{\infty} \frac{d p^{1}}{2 p^{0}} \eta^{2}\left(p^{1}\right)\right\} \eta\left(k^{1}-\pi M\right) \eta\left(q^{1}-\pi M\right)=\frac{1}{4} \frac{\delta}{\delta f\left(k^{1}-\pi M\right)} \\
& \times\left.\frac{\delta}{\delta f\left(q^{1}-\pi M\right)} \exp \left\{\frac{1}{2 \pi} \int_{-\infty}^{\infty} \frac{d p^{1}}{2 p^{0}} f^{2}\left(p^{1}\right)\right\}\right|_{f=0}=(2 \pi)\left|k^{1}-\pi M\right| \delta\left(k^{1}-q^{1}\right),
\end{aligned}
$$

where we have used the relations

$$
\begin{aligned}
\frac{\delta f\left(q^{1}\right.}{\delta f\left(k^{1}\right)} & =(2 \pi) 2 k^{0} \delta\left(k^{1}-q^{1}\right), \\
\frac{\delta f\left(q^{1}-\pi M\right)}{\delta f\left(k^{1}-\pi M\right)} & =(2 \pi) 2\left|k^{1}-\pi M\right| \delta\left(k^{1}-q^{1}\right) .
\end{aligned}
$$


The regularized two-point Wightman functions read

$$
\begin{aligned}
& D^{(+)}(x ; M)=\frac{1}{2 \pi} \int_{-\infty}^{\infty} \frac{d k^{1}}{2 k^{0}}\left(e^{-i k \cdot x}-\cos \left(k^{1} \lambda_{\mathrm{M}}\right)\right) \\
& D^{(-)}(x ; M)=\frac{1}{2 \pi} \int_{-\infty}^{\infty} \frac{d k^{1}}{2 k^{0}}\left(e^{+i k \cdot x}-\cos \left(k^{1} \lambda_{\mathrm{M}}\right)\right) .
\end{aligned}
$$

This testifies the removal of infrared divergences in the quantum field theory of the free massless (pseudo)scalar field in 1+1-dimensional space-time.

\section{Conclusion}

We have shown that the quantum field theory of a free massless (pseudo)scalar field in 1+1-dimensional space-time does not really suffer from an infrared problem. The generating functional of Green functions $Z[J]$ given by $(2.20)$ allowing to calculate any correlation function in the quantum field theory of the free massless (pseudo)scalar field $\vartheta(x)$ does not depend on the infrared cut-off. This occurs due to a simple property of the external source of the free massless pseudoscalar field to have a vanishing integral over the $1+1$-dimensional volume (2.9). The physical meaning of the constraint $(2.9)$ can be easily clarified in the momentum representation, where it corresponds to a removal of a zero-mode configuration of the free massless (pseudo)scalar field $\vartheta(x)$. According to a mechanical analogy of the free massless (pseudo)scalar field $\vartheta(x)$ as a chain of $N$ linear self-coupled harmonic oscillators, a zero-mode configuration of this system describes a motion of a center of mass which does not affect the evolution of the system and can be removed from the system without a loss of information about the response of the system to external perturbations induced by external sources obeying the constraint (2.9).

An analogous treatment of a zero-mode configuration of massless self-coupled scalar fields, described by the $\sigma$-models with internal $O(N)$ symmetry and defined in one and two dimensions, has been suggested by Hasenfratz [21]. Hasenfratz has shown that the exclusion of the zero-mode configuration has allowed to construct a consistent perturbation theory with correct Feynman rules.

By virtue of the constraint (2.9) the infrared cut-off $\mu$ in the generating functional of Green functions (2.4) can be replaced by an arbitrary finite scale $M$. The dependence of $Z[J]$ on a finite scale $M$ allows to regularize the causal two-point Green function of the $\vartheta$-field as well as Wightman functions in the infrared region.

For the regularization of Wightman functions at the level of a redefinition of the $\vartheta$-field we have used the technique of coherent states. We have assumed that the low-frequency quanta of a free massless (pseudo)scalar field are randomized and the wave function of the system of low-frequency quanta can be described as a coherent state. The coherent state of low-frequency quanta depends on a randomized field $\eta\left(k^{1}\right)$ having tangible values only in the close vicinity of zero momenta. Treating this random field as a hidden parameter of an ensemble of low-frequency quanta of the free massless (pseudo)scalar field $\vartheta(x)$ we have assumed that the regularized quantities should be obtained by averaging over the $\eta$-field fluctuations.

Following this prescription we have introduced a new field operator $\vartheta(x ; \eta)$ (3.17) containing translationary invariant quantum contribution to the standard $\vartheta$-field expanded 
into plane waves (1.7). This quantum contribution is proportional to the random $\eta$-field and vanishes when averaged over the $\eta$-field fluctuations. Defining Wightman functions in terms of the new quantum field operators $\vartheta(x ; \eta)$ and averaging over the $\eta$-field fluctuations we have arrived at infrared convergent functions $D^{( \pm)}(x ; M)$ depending on a finite scale $M$. This solves the problem of a consistent, free of infrared divergences, definition of a free massless (pseudo)scalar field in 1+1-dimensional space-time formulated by Klaiber [1] and Coleman [4].

In order to discuss the relation of the free massless (pseudo)scalar field theory free of infrared divergences to the MWH theorem we suggest to calculate the vacuum expectation values of the operators $e^{+i \beta \vartheta(x)}$ and $e^{+i \beta[\vartheta(x)-\vartheta(y)]}$. The calculation runs in the way $[5,12-16]$

$$
\left\langle 0\left|e^{i \beta \vartheta(x)}\right| 0\right\rangle=\left\langle 0\left|e^{i \beta \vartheta(0)}\right| 0\right\rangle=\left.\exp \left\{\beta \frac{\delta}{\delta J(0)}\right\} Z[J]\right|_{J=0}=e^{\frac{1}{2} \beta^{2} i \Delta(0 ; M)} .
$$

The vacuum expectation value of the operator $e^{+i \beta[\vartheta(x)-\vartheta(y)]}$ can be calculated in analogous way and is equal to

$$
\left\langle 0\left|\mathrm{~T}\left(e^{+i \beta[\vartheta(x)-\vartheta(y)]}\right)\right| 0\right\rangle=\left[e^{\frac{1}{2} \beta^{2} i \Delta(0 ; M)}\right]^{2} e^{-\beta^{2} i \Delta(x-y ; M)} .
$$

In the infrared singular quantum field theory of the free massless (pseudo)scalar field $\vartheta(x)$ the causal Green function $i \Delta(0 ; \mu)$ amounts to $[5,12-16]$

$$
i \Delta(0 ; \mu)=-\frac{1}{4 \pi} \ln \left(\frac{\Lambda^{2}}{\mu^{2}}\right)
$$

where $\Lambda$ is the ultra-violet cut-off.

Due to this the correlation function (4.1) in the infrared singular quantum field theory of the free massless (pseudo)scalar field $\vartheta(x)$ should read [5,12-16]

$$
\left\langle 0\left|e^{i \beta \vartheta(x)}\right| 0\right\rangle=\left\langle 0\left|e^{i \beta \vartheta(0)}\right| 0\right\rangle=\lim _{\mu \rightarrow 0}\left(\frac{\mu^{2}}{\Lambda^{2}}\right)^{\beta^{2} / 8 \pi}=0 .
$$

The r.h.s. of (4.4) vanishes for any value of the ultra-violet cut-off $\Lambda$. In turn, the vacuum expectation value of the operator $e^{+i \beta[\vartheta(x)-\vartheta(y)]}$ calculated in the infrared singular quantum field theory of the free massless (pseudo)scalar field $\vartheta(x)$ contains the difference of the Green functions $\Delta(0 ; \mu)-\Delta(x-y ; \mu)$ for which the infrared cut-off $\mu$ cancels itself and the correlation function (4.2) turns out to be independent of $\mu$ and is, therefore, finite in the limit $\mu \rightarrow 0$. This property has been used in the literature [7,13-15] to draw a similarity between the MWH theorem and the infrared singular quantum field theory of the free massless (pseudo)scalar field $\vartheta(x)$.

In the infrared non-singular quantum field theory of the free massless (pseudo)scalar field $\vartheta(x)$ the factor $e^{\frac{1}{2} \beta^{2} i \Delta(0 ; M)}$ is equal to

$$
e^{\frac{1}{2} \beta^{2} i \Delta(0 ; M)}=\lim _{\epsilon \rightarrow 0}\left(-M^{2} \epsilon^{2}+i 0\right)^{\beta^{2} / 8 \pi}=0 .
$$


Therefore, the vacuum expectation values (4.1) and (4.2) vanish simultaneously due to the short-distance behaviour, or differently the ultra-violet divergences. In turn, according to Coleman's analysis of the sine-Gordon model [11] ultra-violet divergences can be removed by renormalization. Following Coleman [11] and removing the common factor (4.5) we obtain the correlation functions

$$
\begin{aligned}
\left\langle 0\left|e^{i \beta \vartheta(0)}\right| 0\right\rangle & =1, \\
\left\langle 0\left|\mathrm{~T}\left(e^{+i \beta[\vartheta(x)-\vartheta(y)]}\right)\right| 0\right\rangle & =\left[-M^{2}(x-y)^{2}+i 0\right]^{-\beta^{2} / 4 \pi} .
\end{aligned}
$$

The most general correlation function of the time-ordered product of the operators $e^{+i \beta \vartheta\left(x_{j}\right)}(j=1, \ldots, n)$ and $e^{-i \beta \vartheta\left(y_{k}\right)}(k=1, \ldots, p)$ given by (2.3) is equal to

$G\left(x_{1}, \ldots, x_{n} ; y_{1}, \ldots, y_{p}\right)=\left\langle 0\left|\mathrm{~T}\left(\prod_{j=1}^{n} e^{+i \beta \vartheta\left(x_{j}\right)} \prod_{k=1}^{p} e^{-i \beta \vartheta\left(y_{k}\right)}\right)\right| 0\right\rangle=$

$=\left.\exp \left\{-i \beta \sum_{j=1}^{n} \frac{\delta}{\delta J\left(x_{j}\right)}+i \beta \sum_{k=1}^{p} \frac{\delta}{\delta J\left(y_{k}\right)}\right\} Z[J]\right|_{J=0}=\left[e^{\frac{1}{2} \beta^{2} i \Delta(0 ; M)}\right]^{n+p}$

$\times \exp \left\{\beta^{2} \sum_{j<k}^{n} i \Delta\left(x_{j}-x_{k} ; M\right)+\beta^{2} \sum_{j<k}^{p} i \Delta\left(y_{j}-y_{k} ; M\right)-\beta^{2} \sum_{j=1}^{n} \sum_{k=1}^{p} i \Delta\left(x_{j}-y_{k} ; M\right)\right\}$.

Removing the ultra-violet divergences by renormalization [11] we arrive at the expression

$$
\begin{aligned}
& G\left(x_{1}, \ldots, x_{n} ; y_{1}, \ldots, y_{p}\right)=\left\langle 0\left|\mathrm{~T}\left(\prod_{j=1}^{n} e^{+i \beta \vartheta\left(x_{j}\right)} \prod_{k=1}^{p} e^{-i \beta \vartheta\left(y_{k}\right)}\right)\right| 0\right\rangle= \\
& =\exp \left\{\beta^{2} \sum_{j<k}^{n} i \Delta\left(x_{j}-x_{k} ; M\right)+\beta^{2} \sum_{j<k}^{p} i \Delta\left(y_{j}-y_{k} ; M\right)-\beta^{2} \sum_{j=1}^{n} \sum_{k=1}^{p} i \Delta\left(x_{j}-y_{k} ; M\right)\right\}= \\
& =\frac{\prod_{j<k}^{n}\left[-M^{2}\left(x_{j}-x_{k}\right)^{2}\right]^{\beta^{2} / 4 \pi} \prod_{j<k}^{p}\left[-M^{2}\left(y_{j}-y_{k}\right)^{2}\right]^{\beta^{2} / 4 \pi}}{\prod_{j=1}^{n} \prod_{k=1}^{p}\left[-M^{2}\left(x_{j}-y_{k}\right)^{2}\right]^{\beta^{2} / 4 \pi}}
\end{aligned}
$$

The most natural way of a simultaneous removal of the ultra-violet divergences is the use of dimensional or analytical regularization allowing to set $\Delta(0 ; M)=0$ [5].

We would like to accentuate that since due to the constraint (2.9) the generating functional of Green functions $Z[J]$ given by (2.14) (or (2.20)) is invariant under the scale transformation $M \rightarrow M^{\prime}$, the correlation functions (4.7) are invariant under the scale transformation $M \rightarrow M^{\prime}$ too. Of course, after the renormalization of ultra-violet divergences carried out at the fixed normalization scale $M$, the correlation functions (4.8) depend on $M$. However, this is normal for any massless quantum field theory, since the correlation functions (4.8) cannot be measured directly. The measurable quantities are the elements of the $S$-matrix. In a free massless (pseudo)scalar field theory the $S$-matrix is trivial and equal to unity $S=1$. The former testifies the independence of the $S$-matrix on the normalization scale $M$. 
The unit value of the vacuum expectation value of the operator $e^{+i \beta \vartheta(0)}$ in (4.6) can be explained as follows. Due to the regularization of the field theory in the infrared region the $\vartheta$-field is not anymore randomized. It does not acquire large classical values proportional to $2 \pi$, as has been pointed out in Refs.[7,15], but varies smoothly around zero, compatible with vacuum fluctuations $\langle 0|\vartheta(0)| 0\rangle=0$.

The time-ordered correlation function of the operator $e^{+i \beta[\vartheta(x)-\vartheta(y)]}$ in $(4.6)$ agrees completely with the results obtained by different authors [12-15] and the results we got in Ref.[5] for the solution of the massless and massive Thirring model with fermion fields quantized in the chirally broken phase. Recall, that the chirally broken phase of the massless Thirring model possesses the ground state [5] coinciding fully with the ground state of the superconducting phase in the Bardeen-Cooper-Schrieffer (BCS) theory of superconductivity [24].

Now let us show that our approach to a quantum field theory of a free massless (pseudo)scalar field $\vartheta(x)$ does not contradict to the MWH theorem. For this aim we suggest to turn to the proof of the MWH theorem expounded in Ref.[13] (see Appendix 4.A, p.219). We have to show that the inequality (A.7) of Ref.[13], transcribed in our notations in continuous Euclidean space for a free massless (pseudo)scalar field theory formulated above, is fulfilled.

In our notations Eq.(A.7) of Ref.[13] can be transcribed in continuous Euclidean space as follows ${ }^{\beta}$

$$
\beta^{2}[\langle 0|\cos \beta \vartheta(0)| 0\rangle]^{2}(-i) \Delta(\vec{\rho} ; M) \leq\langle 0|\sin \beta \vartheta(0) \sin \beta \vartheta(\vec{\rho})| 0\rangle,
$$

where $\vec{\rho}=\left(\rho_{1}, \rho_{2}\right)$ is an infinitesimal 2-dimensional Euclidean vector, $\Delta(\vec{\rho} ; M)$ is a Green function given by

$$
-i \Delta(\vec{\rho} ; M)=\frac{1}{2 \pi} \ln \left(\frac{1}{M \rho}\right)
$$

Here $\rho=\sqrt{\rho_{1}^{2}+\rho_{2}^{2}}$ and $M$ is a finite scale.

The inequality (4.9) is self-consistent. This can be verified in the limit $\beta \rightarrow 0$. In fact, in the limit $\beta \rightarrow 0$ the inequality (4.9) becomes the equality corresponding to the definition of the Wightman function of a free massless (pseudo)scalar field $\vartheta(\vec{\rho})$ in a 2-dimensional Euclidean space.

In order to show this we suggest to divide both sides of (4.9) by $\beta^{2}$. Then, taking the limit $\beta \rightarrow 0$ we arrive at the relation

$$
-i \Delta(\vec{\rho} ; M) \leq\langle 0|\vartheta(0) \vartheta(\vec{\rho})| 0\rangle .
$$

Since by definition $\langle 0|\vartheta(0) \vartheta(\vec{\rho})| 0\rangle=-(1 / 2 \pi) \ln (M \rho)$ is the Wightman function $D(\vec{\rho} ; M)$ of a free massless (pseudo)scalar field $\vartheta(\vec{\rho})$ in 2 -dimensional Euclidean space (2.19), we are able to set $D(\vec{\rho} ; M)=-i \Delta(\vec{\rho} ; M)=-(1 / 2 \pi) \ln (M \rho)$. Therefore, the relation (4.11) should be rewritten in the form of the equality

$$
D(\vec{\rho} ; M)=-i \Delta(\vec{\rho} ; M)=\langle 0|\vartheta(0) \vartheta(\vec{\rho})| 0\rangle .
$$

\footnotetext{
${ }^{3}$ This can be obtained by multiplying the inequality above Eq.(A.7) of Ref.[13] by a factor $e^{i \vec{k} \cdot \vec{\rho}}$ and integrating over $\vec{k}$ at the limit $H \rightarrow 0$, where $H$ is an external magnetic field.
} 
This confirms the self-consistency of the inequality (4.9).

According to Itzykson and Drouffe [13] the vacuum expectation value $\langle 0|\cos \beta \vartheta(0)| 0\rangle$ should be identified with the spontaneous magnetization $\mathcal{M}$

$$
\mathcal{M}=\langle 0|\cos \beta \vartheta(0)| 0\rangle .
$$

The vacuum expectation value of the r.h.s. of (4.9) is equal to

$$
\langle 0|\sin \beta \vartheta(0) \sin \beta \vartheta(\vec{\rho})| 0\rangle=\frac{1}{2} e^{\beta^{2} i \Delta(0 ; M)}\left[e^{-\beta^{2} i \Delta(\vec{\rho} ; M)}-e^{+\beta^{2} i \Delta(\vec{\rho} ; M)}\right] .
$$

Using (4.1) for the calculation of the spontaneous magnetization $\mathcal{M}$ and Eqs.(4.13) and (4.14) we recast the inequality (4.9) into the form

$$
e^{\beta^{2} i \Delta(0 ; M)} \ln \left(\frac{1}{M \rho}\right) \leq \frac{\pi}{\beta^{2}} e^{\beta^{2} i \Delta(0 ; M)}\left[(M \rho)^{-\beta^{2} / 2 \pi}-(M \rho)^{\beta^{2} / 2 \pi}\right] .
$$

Renormalizing $\langle 0|\cos \beta \vartheta(0)| 0\rangle$ and $\langle 0|\sin \beta \vartheta(0) \sin \beta \vartheta(\vec{\rho})| 0\rangle$, i.e. removing the constant divergent factors $e^{\beta^{2} i \Delta(0 ; M)}$ in both sides of (4.15) and getting $\mathcal{M}=1$, we obtain

$$
\ln \left(\frac{1}{M \rho}\right) \leq \frac{\pi}{\beta^{2}}\left[(M \rho)^{-\beta^{2} / 2 \pi}-(M \rho)^{\beta^{2} / 2 \pi}\right] .
$$

This inequality is always fulfilled for $\rho \rightarrow 0$ as required by the derivation of the MWH theorem according to Ref.[13]. Thus, the renormalized spontaneous magnetization $\mathcal{M}=$ 1 , obtained in our approach as a vacuum expectation value of the operator $\cos \beta \vartheta(0)$, agrees with the inequality (4.9), which is a continuous analogy of the lattice inequality used for the derivation of the MWN theorem in [13]. We notice that the renormalized spontaneous magnetization $\mathcal{M}=1$, being the observable quantity, does not depend on the normalization scale $M$.

It is seen that in the limit $\beta \rightarrow 0$ the relation (1.16) becomes an equality. This testifies the validity of our statement given by (4.12).

We would like to emphasize that our result, $\mathcal{M}=1$, does not contradict to the $\mathrm{MWH}$ theorem, since it goes beyond the scope of the applicability of the MWH theorem [10]. As has been pointed out by the authors [10], the vanishing of the long-range order can be inferred only for non-zero temperature $T \neq 0[10]$ and none conclusion about its value can be derived for $T=0$ [10]. Since spontaneous magnetization and fermion condensation have been found in the free massless (pseudo)scalar field theory, discussed above, and the massless Thirring model in Ref.[5] at $T=0$, these results cannot be considered as counterexamples to the MWH theorem.

\section{Acknowledgement}

This work was supported in part by Fonds zur Förderung der Wissenschaftlichen Forschung P13997-TPH. 


\section{References}

[1] B. Klaiber, in LECTURES IN THEORETICAL PHYSICS, Lectures delivered at the Summer Institute for Theoretical Physics, University of Colorado, Boulder, 1967, edited by A. Barut and W. Brittin, Gordon and Breach, New York, 1968, Vol. X, part A, pp.141-176.

[2] W. Thirring, Ann. Phys. (N.Y.) 3, 91 (1958).

[3] V. Glaser, Nuovo Cim. 9, 990 (1958); W. Thirring, Nuovo Cim. 9, 1007 (1958).

[4] S. Coleman, Comm. Math. Phys. 31, 259 (1973).

[5] M. Faber and A. N. Ivanov, Eur. Phys. J. C 20, 723 (2001), hep-th/0105057.

[6] J. Goldstone, Nuovo Cimento 19, 154 (1961); J. Goldstone, A. Salam, and S. Weinberg, Phys. Rev. 127, 965 (1962).

[7] C. Itzykson and J.-B. Zuber, in QUANTUM FIELD THEORY, McGraw-Hill Book Company, New York, 1980, p.525.

[8] (see [7], pp.519-521)

[9] John R. Klauder and Bo-Sture Skagerstam, in COHERENT STATES, Applications in Physics and Mathematical Physics, World Scientific, Singapore, 1985; John W. Negele and Henri Orland, in QUANTUM MANY-PARTICLE SYSTEMS, Addison-Wesley Publishing Co., New York, 1988;(see [7], pp.118-120).

[10] N. D. Mermin and H. Wagner, Phys. Rev. Lett. 17, 1133 (1966); P. C. Hohenberg, Phys. Rev. 158, 383 (1967); N. D. Mermin, J. Math. Phys. 8, 1061 (1967).

[11] S. Coleman, Phys. Rev. D 11, 2088 (1975).

[12] J. Fröhlich and P. A. Marchetti, Comm. Math. Phys. 116, 127 (1988).

[13] C. Itzykson and J.-M. Drouffe, in STATISTICAL FIELD THEORY, From Brownian motion to renormalization and lattice gauge theory, Vol. I, Cambridge University Press, Cambridge, 1989, pp.219-224.

[14] E. Abdalla, M. C. B. Abdalla, and K. D. Rothe, in Non-perturbative methods in 2 DIMENSIONAL FIELD THEORY, World Scientific, Singapore, 1991; J. Zinn-Justin, in QUANTUM FIELD THEORY AND CRITICAL PHENOMENA, Clarendon Press - Oxford, 1993.

[15] K. Huang, in QUANTUM FIELD THEORY, From Operators to Path Integrals, John Willey \& Sons, Inc., New York, 1998, pp.363-367.

[16] M. Faber and A. N. Ivanov, On the solution of the massless Thirring model with fermion fields quantized in the chiral symmetric phase, IK-TUW-Preprint 0111401, hep-th/0112183. 
[17] J. Schwinger in PARTICLES AND SOURCES, Gordon and Breach, New York 1969 and PARTICLES, SOURCES AND FIELDS, Addison-Wesley Publishing Co., Massachusetts 1970 .

[18] P. M. Morse and H. Feshbach, in METHODS OF THEORETICAL PHYSICS, McGraw-Hill Book Company, New York-Toronto-London, 1953; I. M. Gel'fand and G. E. Shilov, in GENERALIZED FUNCTIONS, Properties and Operations, Vol. I, Academic Press, New York and London, 1964; G. Arfken, in MATHEMATICAL METHODS FOR PHYSICISTS, Academic Press, New York and London, 1966.

[19] T. H. R. Skyrme, Proc. Roy. Soc. A 247, 260 (1958); ibid. A 262, 237 (1961).

[20] M. Faber, Few Body System, 30, 149 (2001), hep-th/9910221.

[21] P. Hasenfratz, Phys. Lett. B 141, 385 (1984).

[22] (see [15] pp.1-7); A. L. Fetter and J. D. Walecka, in THEORETICAL MECHANICS OF PARTICLES AND CONTINUA, McGraw-Hill Publishing Co., New York, 1980, pp.101-130; J. C. Slater and N. H. Frank, in MECHANICS, McGraw-Hill Book Co.,Inc., New York and London, 1947, pp.21-40 and pp.122-161; H. Goldstein, in CLASSICAL MECHANICS, Addison-Wesley Publishing Co., Inc., Reading, Massachusetts, USA, London, England, 1950, pp.318-372; L. D. Landau and E. M. Lifshitz, in MECHANICS, Pergamon Press, Oxford-London-Paris, 1960, pp.58-74.

[23] HANDBOOK OF MATHEMATICAL FUNCTIONS, with Formulas, Graphs, and Mathematical Tables, ed. by M. Abramowitz and I. E. Stegun, U.S. Department of Commerce, National Bureau of Standards, Applied Mathematics Series • 55, 1972, p.376 formulas (9.6.21) and (9.6.24).

[24] J. Bardeen, L. N. Cooper and J. R. Schrieffer, Phys. Rev. 106, 162 (1957); Phys. Rev. 108, 1175 (1957). 